\title{
Ethischer Organstreit? Der schwierige Weg von der Klimaethik zur Klimapolitik
}

Klimapolitik gilt heute im globalen Maßstab sehr wohl als ein wichtiger Politikbereich, doch entstehen im Blick auf die tatsächliche Zielerreichung innerhalb dieser policy mehr und mehr Fragen. So klar und deutlich die Aussagen vieler Klimaexperten formuliert werden und so drastisch das Debatten-Vokabular auch sein mag - neben den eher neutralen „Klimawandel“ drängen sich stets auch Begriffe wie „Klimakatastrophe“ oder „Klimakrieg“-, deutliche Effekte auf eine „klimafreundliche" Politikformulierung sind noch kaum zu verzeichnen. Auch die auf den diversen „Klimagipfeln“ getroffenen Vereinbarungen sind nicht immer das Papier wert, auf denen sie unterzeichnet werden (und nicht nur deshalb sind sie vielleicht eher ein Teil des Problems als ein Teil der Lösung).

So ist im Dezember vergangenen Jahres Kanada aus dem Kyoto-Protokoll ausgestiegen; inhaltliches Hauptargument für den Ausstieg waren die drohenden Strafabgaben für die Nicht-Einhaltung von Klimazielen. Diese Episode verdeutlicht zweierlei: zum einen die geringe Bindekraft aktueller Beschlüsse und Konventionen zum Klimaschutz, zum anderen die Präge- und Durchsetzungskraft nationaler Debatten und parlamentarischer Mehrheitsverhältnisse in einem per se global konstruierten Politikfeld. Die Argumentation fiel Peter Kent, dem kanadischen Umweltminister, leicht: Da die Großverschmutzer China und USA das Kyoto-Protokoll nicht unterzeichnet hätten, sei der gesamte Prozess zum Scheitern verurteilt. ${ }^{1}$ Selbstverständlich sind die Etablierung eines weltweiten Klimadiskurses und die allmähliche Entwicklung eines globalen Steuerungssystems aus Gipfeltreffen, Protokollen und konkreten Mechanismen zur Zielerreichung wichtige Schritte auf dem Weg zu einer erfolgreichen Klimapolitik - doch ganz offenbar reichen sie nicht aus, um einen tatsächlichen Politikwandel zu vollziehen und die produktive Formulierung einer klimaverträglichen Politik zu gewährleisten.

An dieser Stelle gerät das Feld der Klimaethik in den Blick, die sich zunächst einmal vehement gegen die naturwissenschaftlich-ökonomisch geprägten ,,management approaches“" herkömmlicher Klimapolitik wendet. Dale Jamieson, Philosoph

1 Das Statement von Umweltminister Peter Kent ist online einsehbar unter http://www.ec.gc.ca/defa ult.asp?lang=En\&n=FFE36B6D-1\&news=6B04014B-54FC-4739-B22C-F9CD9A840800 (Stand: 29.2.2012). 
und Umweltforscher an der New York University, betont die Rolle ethischer Positionen und Perspektiven in der Debatte um den Klimawandel: „The problem that we face is not a purely scientific problem that can be solved by the accumulation of scientific information. Science has alerted us to a problem, but the problem also concerns our values. It is about how we ought to live and how humans should relate to one another and to the rest of nature." (Jamieson 2010: 79) In seiner Bewertung der Debatte um eine Politikformulierung angesichts von Erderwärmung und Klimawandel erteilt er technizistisch-ökonomischen Management-Ansätzen eine Absage. Jamieson fügt eine ethische Dimension hinzu und erinnert an Werte, Charakter und die Fähigkeit, durch individuelles Handeln Dinge verändern zu können - selbst wenn es viel Zeit braucht und die Effekte kaum spürbar sind: „When we „economize" our behaviour in the way that is required for calculating, we systematically neglegt the subtle and indirect effects of our actions, and for this reason we see individual action as inefficacious.“ (84). ${ }^{2}$

Diese Grundargumentation durchzieht auch den jungen deutschen Zweig der Debatte, die durchaus zuversichtlich wirkt. Bernward Gesang, Professor für Wirtschaftsethik in Mannheim, notiert in seiner Monografie zur Klimaethik: „Die Erfahrung lehrt, dass wir uns aus vielen scheinbar aussichtslosen Situationen befreien konnten. Wir sollten uns hier nicht unterschätzen. Zumal das Problem weder technisch schwer zu lösen noch eine Lösung besonders teuer ist. Jeder Einzelne kann zu einer Lösung beitragen, insbesondere indem er oder sie politisch aktiv wird." (2011: 222 f.) Der Greifswalder Philosoph und Umweltethiker Konrad Ott betont dabei ebenfalls die Rolle der Bürger, die den politischen Handlungsdruck steigern könne: „Es könnte sogar sein, dass die Menschheit sich derzeit in dem entscheidenden ,window of opportunity“ befindet und es deswegen gerade jetzt auf politischen Gestaltungswillen besonders ankommt. Die Zivilgesellschaft sollte die Politik nicht aus der Pflicht zur Einigung entlassen.“ (Ott 2009: 74)

Der Berliner Publizist Josef Bordat bringt schließlich explizit die Aktionsräume des politischen Systems ins Spiel, die allerdings nur bedingt geeignet scheinen, klimaethische Impulse aufgreifen und umsetzen zu können: „Die Organisation „VN“ [Vereinte Nationen] übernimmt mit der Institution „Kyoto“ Verantwortung in Zeiten des Klimawandels. Schon dieses Beispiel macht die Krux der Institutionenethik auf globaler Ebene deutlich: Die Organisation (die VN) müsste neben dem ,guten Willen" zur Institution (dem völkerrechtlichen Vertrag) auch die faktische Durch-

2 Eine detailliertere Darstellung der Debatte um die Klimaethik ist an dieser Stelle nicht möglich; vergleiche dazu einführend Gardiner u. a. 2010, Arnold 2011 sowie den Forums-Beitrag von Marco Iorio. 
setzungsmöglichkeit haben, um die Institution in der Rechtspraxis wirksam werden zu lassen." (Bordat 2010: 193)

Man muss nicht allein die globale Perspektive und ein auf dieser Ebene besonders verwobenes Akteursgeflecht in den Blick nehmen, um die Problematik einer praktischen Implementierung klimaethischer Positionen und Überlegungen zu erkennen. Zwar hat die globale „climate governance“ inzwischen eine ganze Reihe neuer Institutionen, Organisationen und Verfahren ausgebildet, doch funktioniert dieses „Klimaregime“ keineswegs fehlerfrei - und wie das kanadische Beispiel zeigt, ist die „Wirkungsmacht“ gegenüber den Handlungen klassischer nationaler PolitikAkteure sehr begrenzt. Dennoch kann Ethik sehr wohl als ein Instrument modernen Politikmanagements verstanden werden: Die stärkere Präsenz und die Einbindung der „Zivilgesellschaft“ in die öffentliche Debatte verankert das Thema dauerhaft auf der Agenda von Parteien, Parlamenten und Regierungen. Doch als zentrale Frage bleibt: Wie können politische Akteure die Impulse der Auseinandersetzung mit Ethik, Gerechtigkeit und Verantwortung im Klimawandel produktiv in konkrete Arbeitsprozesse integrieren? Und wie lässt sich daraus ein „klimaethisches Entscheidungshandeln" realisieren?

Interessanterweise liefert die viel gescholtene Ethik-Kommission Sichere Energieversorgung im Umfeld der sogenannten „Energiewende“ ein anschauliches Beispiel für einen neuartigen Prozess der Einbettung klimaethischer Positionen in die politische Praxis. Das gilt trotz (oder gerade wegen?) der weitgehend misslungenen Ausgestaltung der Kommission als ,ethisches Beratungsorgan“(vgl. Kettner 2005), an dem die Lernfähigkeit politischer Institutionen und Prozesse deutlich wird.

\section{Von der Klimaethik zur Klimapolitik?}

Inzwischen sind zahlreiche neue Impulse für „ethische Beratungsorgane“ entstanden, darunter eben auch die „Klimaethik“, die insbesondere die etablierten Politikfelder Umwelt- und Energiepolitik adressiert. Die dabei formulierten Überlegungen und Empfehlungen fügen dem (natur-)wissenschaftlich ermittelten Wissen über den Klimawandel meist eine Perspektive auf „Klimagerechtigkeit“ hinzu. Gesang vertritt dabei den Standpunkt, dass diese Form der Herangehensweise zum Scheitern verurteilt ist, da sich die unterschiedlichen Gerechtigkeitskonzeptionen nicht oder nur unter großen Schwierigkeiten miteinander vereinbaren ließen (vgl. Gesang 2011: 47 ff.). Gesang postuliert dagegen ein utilitaristisches Prinzip der Klimaethik, das auf den künftigen Nutzen politischer Maßnahmen zum Klimaschutz abzielt. Für die tatsächliche Formulierung einer ,ethisch entwickelten“ Klimapolitik erscheint dabei die Abwägung zwischen dem „Nutzenpotenzial der Zukunft“ und den „Kosten 
der Gegenwart“ (146) zentral. Denn genau an dieser Stelle setzen konkrete Handlungen und Entscheidungen politischer Akteure an: Wenn zum Beispiel ein Land entscheidet, dass die Gefahr in der Gegenwart zu leistender Strafzahlungen höher gewichtet wird als der künftige Nutzen der Erreichung von Klimazielen - und dann im parlamentarischen Verfahren eine bereits geleistete Ratifizierung zurückgezogen wird.

Das konkrete Beispiel Kanadas macht deutlich, dass die Modelle der Klimaethik individuelles und gesellschaftliches Handeln vorerst nur anleiten und vorstrukturieren können. Allerdings entstehen dabei nur ,virtuelle Handlungskorridore“ in einem diskursiven Raum - die Entscheidungspolitik bleibt bis auf weiteres die Domäne politischer Akteure. Dieser Befund ist jedoch nur auf den ersten Blick ernüchternd, denn durch den dauerhaften und sich ausweitenden Diskurs um die Klimaethik kommt es auch im politischen Entscheidungszentrum zu gelegentlichen Ethik-Experimenten. Und selbst wenn diese Experimente bei näherem Hinsehen als „Vorerst gescheitert" gelten müssen, so resultieren daraus doch Ansätze für politisches Lernen. Das zeigt - auf irritierend-gelungene Weise - das Politikmanagement im Umfeld der Änderung des deutschen Atomgesetzes. „Deutschlands Energiewende“, dieses „Gemeinschaftswerk für die Zukunft“ wurde auch unter Bezugnahme auf ethische Positionen realisiert. ${ }^{3}$ Allerdings hat dieser Politikprozess einen Haken - die so genannte „Ethik-Kommission“ hat gleich in mehrfacher Hinsicht die Anforderungen an ein ethisches Beratungsorgan nicht erfüllen können.

\section{Ethisches Organversagen?}

Die Ethik-Kommission „Sichere Energieversorgung“ wurde im März 2011 auf Beschluss der Bundeskanzlerin eingerichtet und hatte mit der konstituierenden Sitzung am 4. April 2011 ihre Arbeit aufgenommen. Zentrale Bestandteile der Kommissionsarbeit waren mehrere Klausurtagungen sowie ein „Öffentlicher Dialog“, bei dem am 28. April 2011 zusätzliche Experten und Verbandsvertreter angehört wurden. Der Abschlussbericht der Kommission wurde am 30. Mai 2011 vorgelegt. ${ }^{4}$ Der auch in der breiten Öffentlichkeit vorgetragenen Kritik an der personellen Zusammensetzung der Kommission (Goltz 2011) ist eine abstrakte, sich aus der wissen-

3 Im Titel des Abschlussberichts der Ethik-Kommission „Deutschlands Energiewende - Ein Gemeinschaftswerk für die Zukunft“ - ist der Hinweis auf eine „,zukünftige Nutzenorientierung“ deutlich zu erkennen.

4 Vergleiche hierzu die Mitschrift der Pressekonferenz zur Erklärung von Bundeskanzlerin Merkel am 22.3.2011 (http://www.bundesregierung.de/Content/DE/Mitschrift/Pressekonferenzen/ 2011/03/2011-03-22-statements-kernenergie-in-deutschland.html; Stand 29.2.2012) sowie den abschließenden Kommissionsbericht (Ethik-Kommission Sichere Energieversorgung 2011). 
schaftlichen Auseinandersetzung mit „ethischen Beratungsorganen“ (EBOs) speisende Perspektive hinzuzufügen. Hilfreich sind dafür die von Kettner entwickelten „Regelgruppen“, die zugleich den Arbeitsprozess ethischer Beratungsorgane nachzeichnen: Institutionalisierungsregeln, Input-Regeln, Regeln der Deliberation sowie Output-Regeln (2005:12 f.).

Im Falle der Ethik-Kommission Sichere Energieversorgung wurden bereits im Vorgang der Einrichtung verschiedene Basisannahmen und Grundregeln ignoriert; so erfolgten bereits die Implementierung des Gremiums sowie die Rekrutierung der Mitglieder weitgehend intransparent. Konkrete Verfahrensabläufe wurden lediglich im Rahmen der öffentlichen Anhörung sichtbar, darüber hinaus war jedoch wenig über die Entscheidungsvorbereitung und -findung zu erfahren. Der Schlussbericht verkündet lediglich die Resultate der Kommissionsarbeit, wobei unmittelbar der geringe Stellenwert von ethischen Positionen und Argumentationen im Vergleich zu der dominierenden Ebene der politikfeldbezogenen Aussagen und Handlungsempfehlungen auffällt. Diese Praxis steht dabei im Widerspruch zu den Anforderungen an genuine EBOs, die nach Kettner „keine Black Boxes [sind], bei denen man nicht weiß, was zwischen Input und Output geschieht." (ebd.) Rippe stellt in diesem Zusammenhang ebenfalls eindeutig fest: „Auch Expertenkommissionen sind eine öffentliche Angelegenheit. Das bedeutet, daß die Öffentlichkeit Zugang zu allen Sitzungen haben, Protokolle einsehen können und die Möglichkeit haben sollte, auf die Diskussion zurückzuwirken.“ (2000: 162)

Eine detaillierte Gegenüberstellung der Kommissionspraxis mit einer diskursethisch grundierten Modellierung ethischer Beratungsorgane ist an dieser Stelle nicht möglich, dennoch soll zumindest auf einen weiteren auffälligen Befund hingewiesen werden: Eine zentrale Leistung der Ethik-Beratung besteht auch in der fallbezogenen Diskussion und Abwägung moralischer Stellungnahmen, die für möglichst viele Menschen zugänglich sein sollen. Sehr anschaulich gelöst wird dieser Anspruch etwa in den Arbeiten des Deutschen Ethikrates, die zu diesem Zweck zunehmend auch responsive Formate (z. B. Befragungen, Online-Diskurse) einbinden. Auch in dieser Hinsicht bleibt die Ethik-Kommission Sichere Energieversorgung hinter den selbst formulierten Ansprüchen zurück, da in der Kommissionsarbeit keine Explizierung ethischer Dilemmata im Prozess einer energiepolitischen Politikformulierung stattgefunden hat und nur minimale Möglichkeiten für eine Anschlusskommunikation nach der Vorstellung des Abschlussberichts gegeben waren.

In formaler Hinsicht war die Arbeit der Ethik-Kommission zwar in den parlamentarischen Prozess integriert, doch wurde die zeitliche Nähe zur Reaktor-Katastrophe in Fukushima (11. März 2011) und dem „Atom-Moratorium“ mit der kurz- 
fristigen Abschaltung der Atomkraftwerke (15. März 2011) stets als Ansatzpunkt für eine Kritik des nur eine Woche später durch die Bundeskanzlerin eingesetzten Gremiums genutzt. Die Einbettung in den politischen Prozess erfolgte mit Verweis auf die Prüfungen durch die Ethik-Kommission und die Reaktorsicherheitskommission, um nach deren Abschluss eine formale Änderung des Atomgesetzes zu erwirken. ${ }^{5}$ Durch diese Vorgeschichte relativierte sich auch die Wirkungskraft der Ethik-Kommission, der „Beratungsimpuls“ wurde regierungsseitig in den parlamentarischen Prozess eingespeist und spielte bei der Debatte um die angestrebte Gesetzesänderung keine besondere Rolle. ${ }^{6}$ Durch das Atom-Moratorium, breite Proteste gegen die Nutzung der Atomenergie durch die Bevölkerung und nicht zuletzt die Etablierung des Atomausstiegs als zentrales Wahlkampfthema in BadenWürttemberg war die politische Sachentscheidung vorstrukturiert worden, noch bevor die Kommission überhaupt ihre Arbeit aufgenommen hatte.

\section{Wohin mit der Ethik?}

Das Fallbeispiel der Ethik-Kommission Sichere Energieversorgung gerät somit zu einer Illustration für die Attraktivität, Ethik als zusätzliche Ressource in den politischen Prozess zu integrieren. Die skizzierten Erfahrungen machen deutlich, dass die Ethik-Orientierung von Beratungsorganen mit eindeutigem Politikfeld-Bezug noch mit großen Schwierigkeiten in der praktischen Umsetzung behaftet ist. Dennoch liegen im kreativen Umgang mit neuen Formaten große Chancen für die nächsten Schritte auf dem Weg zu einer wirksamen Politik im Klimawandel. Derartige Kommissionen können als ein neuartiges Bindeglied zwischen naturwissenschaftlich-technischer Klimaforschung, einer philosophisch hergeleiteten Klimaethik und praktischer Politik fungieren. Im Unterschied zu klassischen Akteuren der wissenschaftlichen Politikberatung ${ }^{7}$ können Ethik-Kommissionen zusätzliche Expertisen in den Politikprozess integrieren und für eine Verbreiterung der Debatte sorgen. Vor allem aber docken sie an einer anderen Stelle an den Politikbetrieb an: Sie sind näher am parlamentarischen Prozess verortet und verfügen daher - zumin-

5 Die entsprechende Gesetzesänderung wurde am 30. Juni 2011 mit breiter Mehrheit im Bundestag beschlossen, am 8. Juli stimmte der Bundesrat der Änderung zu. Nach der Unterzeichnung des Gesetzes durch Bundespräsident Wulff trat das neue Atomgesetz schließlich am 8. August 2011 in Kraft.

6 Vergleiche dazu die Dokumentation der Debatte im Plenarprotokoll Nr. 17/117 vom 30. Juni 2011. Darin überwiegen kritische Erwähnungen der Kommission, auf von ihr erarbeitete Empfehlungen wird nicht eingegangen, die ethische Dimension der Energiewende bleibt nahezu vollständig unerwähnt.

7 Im Falle des Klimawandels ist hier vor allem der „Wissenschaftliche Beirat der Bundesregierung Globale Umweltveränderungen“(WBGU) zu nennen (vgl. www.wbgu.de). 
dest theoretisch - über andere Instrumente der Einflussnahme, die stärker auf den Bereich der Deliberation und Entscheidungsvorbereitung von Abgeordneten zielen. Damit sie ihre Wirkungskraft voll entfalten können und auch größere Öffentlichkeiten als nur den Plenarsaal erreichen, müssen künftig jedoch die besonderen Anforderungen ethischer Beratungsorgane beachtet werden. Das komplexe Problem des Klimawandels benötigt ganz offenbar auch komplexe institutionelle Arrangements, in denen Ethik-Akteure durchaus ihren Platz finden können. Man darf jedoch nicht davon ausgehen, dass deren Einbettung allein vernunft- und verantwortungsgeleitet vonstatten gehen wird.

\section{Literatur}

Arnold, Denis G. (Hrsg.), 2011: The Ethics of Global Climate Change, Cambridge. Bordat, Josef, 2010: Ethik in Zeiten des Klimawandels, in: Martin Voss (Hrsg.), Der Klimawandel. Sozialwissenschaftliche Perspektiven, Wiesbaden, 189-204. Ethik-Kommission Sichere Energieversorgung, 2011: Deutschlands Energiewende - Ein Gemeinschaftswerk für die Zukunft, Berlin.

Gardiner, Stephen M./Caney, Simon/Jamieson, Dale/Shue, Henry (Hrsg.), 2010: Climate Ethics. Essential Readings, Oxford.

Gesang, Bernward, 2011: Klimaethik, Berlin.

Goltz, Astrid, 2011: Öffentliche Anhörung der Ethikkommission. Online-Publikation unter http://blog.campact.de/2011/04/offentliche-anhorung-der-ethikkommission/ (Stand: 29.2.2012).

Jamieson, Dale, 2010: Ethics, Public Policy, and Global Warming, in: Stephen M. Gardiner/Simon Caney/Dale Jamieson/Henry Shue (Hrsg.): Climate Ethics. Essential Readings, Oxford, 77-86.

Kettner, Matthias, 2005: Ethik-Komitees. Ihre Organisationsform und ihr moralischer Anspruch, in: Erwägen, Wissen, Ethik 16 (1), 3-16.

Ott, Konrad, 2009: Grundzüge der Klimaethik, in: Architektenkammer NordrheinWestfalen (Hrsg.), Natur und gebaute Umwelt, Düsseldorf, 57-64.

Rippe, Klaus-Peter, 2000, Ethikkommissionen in der deliberativen Demokratie, in: Matthias Kettner (Hrsg.), Angewandte Ethik als Politikum, Frankfurt a. M., 140-164. 
Korrespondenzanschrift:

Prof. Dr. Christoph Bieber

Welker-Stiftungsprofessur für Ethik in Politikmanagement und Gesellschaft NRW School of Governance

Institut für Politikwissenschaft

Universität Duisburg-Essen

Lotharstraße 53

47057 Duisburg

E-Mail: christoph.bieber@uni-duisburg-essen.de

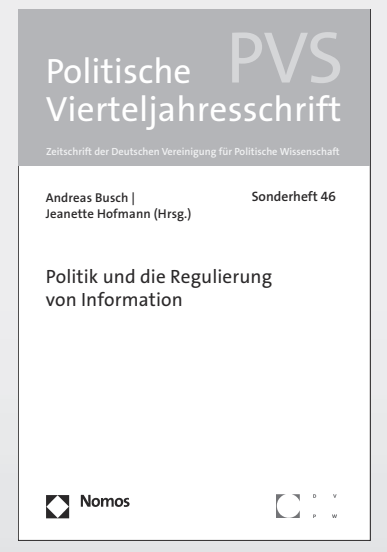

\section{Politik und die Regulierung von Information}

Sonderheft PVS 46

Herausgegeben von Andreas Busch und Jeanette Hofmann

2012, 514 S., brosch., 49,90€

ISBN 978-3-8329-6540-2

Die Entwicklung zur „Informationsgesellschaft“ und die zunehmende Digitalisierung haben große wirtschaftliche Dynamiken freigesetzt, aber gleichzeitig auch neue politische und soziale Konflikte geschaffen. Das Ziel dieses Sonderbandes der Politischen Vierteljahresschrift ist es, diese Umbrüche in empirischer und theoretischer Hinsicht zu analysieren.

Weitere Informationen:

www.nomos-shop.de/13557 